\title{
OUTCOME OF PATIENTS TREATED WITH BLADDER PRESERVATION PROTOCOL IN UROTHELIAL CARCINOMA OF URINARY BLADDER
}

\author{
Abdus Samad Syed, Sameed Hussain, Fouzia Abdus Samad* \\ Combined Military Hospital/National University of Medical Sciences (NUMS) Rawalpindi Pakistan, *Fauji Foundation Hospital, Rawalpindi Pakistan
}

\begin{abstract}
ABSTARCT
Objective: To explore trimodality treatment with neoadjuvant chemotherapy followed by concurrent chemoradiotherapy as an alternative approach to neoadjuvant chemotherapy followed by radical cystectomy, for the treatment of non-metastatic muscle invasive bladder carcinoma.

Study Design: Retrospective observational study.

Place and Duration of Study: Combined Military Hospital Rawalpindi Pakistan, from 2006 and 2015.

Methodology: A total of 122 patients were evaluated in a retrospective manner. Primary endpoint was overall survival. Patients received four courses of neoadjuvant chemotherapy followed by radical concurrent chemoradiotherapy with Cisplatin as radiation sensitizer.

Result: 5-year overall survival was $80(66 \%)$ in this population and a complete response following completion of treatment was seen in $93(76.3 \%)$ patients. Subset analysis showed markedly increased 5-year overall survival of around $104(85 \%)$ in patients having complete response after neoadjuvant chemotherapy.

Conclusion: The study concludes that bladder preservation is an acceptable alternative to radical cystectomy in selected population especially among those who had a complete response to the initial four courses of chemotherapy.
\end{abstract}

Keywords: Bladder preservation, Concurrent chemoradiotherapy, Organ preservation, Radical cystectomy, Urinary bladder carcinoma.

How to Cite This Article: Syed AS, Hussain S, Samad FA. Outcome of Patients Treated with Bladder Preservation Protocol in Urothelial Carcinoma of Urinary Bladder. Pak Armed Forces Med J 2021; 71(6): 2118-2121. Doi: https://doi.org/10.51253/pafmj.v71i6.5847

This is an Open Access article distributed under the terms of the Creative Commons Attribution License (https://creativecommons.org/licenses/by-nc/4.0/), which permits unrestricted use, distribution, and reproduction in any medium, provided the original work is properly cited.

\section{INTRODUCTION}

The standard of care in muscle invasive bladder carcinoma is radical cystectomy and has been so, for last 50 years or more. The 5-year survival rate has been between $50-70 \% .{ }^{1}$ A recent analysis at a single institute regarding radical cystectomy showed that at median follow up time of 65 months, the survival is about $67 \% .^{2}$ Need of alternatives for the radical cystectomy have always been actively persuaded due to two main reasons. First is that the long-term survival rates with radical cystectomy have never been significantly superior to bladder preservation. Secondly, the long-term cost of radical cystectomy is very high as compared to bladder preservation especially when expertise for continent diversion is not widely available in most centres in Pakistan. Urostomy with ileal conduit formation may lead to disturbing psychological impact as patient is socially affected.

Bladder preservation protocols are aimed not only to improve the quality of life but also to improve the overall survival. Upfront concurrent chemoradiotherapy (CCRT) has shown equivalent results to

Correspondence: Dr Abdus Samad Syed, Department of Radiation Oncology, Combined Military Hospital Rawalpindi Pakistan

Received: 13 Dec 2020; revision received: 06 May 2021; accepted: 01 Jun 2021 radical cystectomy but has not been accepted as the standard of care so far. ${ }^{3-5}$ Neoadjuvant chemotherapy followed by CCRT has been commonly used in the past. ${ }^{6-8}$ Neoadjuvant chemotherapy results, not only in reducing the chances of distant metastasis but also improves local control.9,10 Firstly, this is achieved by killing the cancer cells in an early stage of disease, as they would be less in number at that point in time. Secondly, the hypoxic cells that are resistant to radiation would be more sensitive to chemotherapy combined with radiotherapy9. Third, drug resistance in cancer cells is related to spontaneous mutations, which increases with the increasing number of cancer cells. ${ }^{9}$ So an early use of chemotherapy followed by CCRT is expected to show better results than radical cystectomy or upfront CCRT. This study was designed to study the impact of this approach on overall survival in patients with muscle invasive bladder carcinoma.

\section{METHODOLOGY}

A retrospective analysis of patients treated with concurrent chemoradio-therapy, from 2006 to 2015, at Radiation Oncology Department, Combined Military Hospital Rawalpindi was carried out. Patients declining radical cystectomy or in whom radical cystectomy could not be performed due to co-morbid conditions or 
age; but were fulfilling the inclusion criteria were included into the study after Ethical Review Board approval (127/11/20).

Inclusion Criteria: Patients having histopathologically proven muscle invasive urothelial carcinoma of the bladder, AJCC T stage T2-T4, N0-N1, ECOG status 0-2, $\mathrm{Hb}>10 \mathrm{~g} / \mathrm{dL}, \mathrm{TLC}>4000 / \mathrm{mm}^{3}$, Platelets $>150000 /$ $\mathrm{mm}^{3}$, normal serum urea, creatinine, electrolytes and, liver function tests were included in the study.

Exclusion Criteria: Patients with prior history of chemotherapy, radiotherapy or any other malignancy or pregnancy were excluded from the study.

A total of 122 eligible patients were evaluated. Non-probability consecutive sampling technique was used.

Before the start of therapy, blood $\mathrm{CP}$, liver function tests, serum urea, creatinine, serum sodium and potassium and CT scan or MRI of pelvis were carried out. Maximal transurethral resection of bladder tumor was performed to remove as much tumor as possible from bladder and the specimen sent for histopathological confirmation of the carcinoma. After histopathological confirmation of malignancy, baseline and metastatic work-up included haematological and biochemical blood tests and imaging of chest, abdomen and upper urinary tract.

Neoadjuvant chemotherapy with Gemcitabine and Cisplatin was initiated. Full blood counts, serum urea, creatinine and liver function tests were repeated before each course of chemotherapy. Gemcitabine in a dose of $1200 \mathrm{mg} / \mathrm{m}^{2}$ was administered on day 1 and day 8. Cisplatin was administered on day 1 at $75 \mathrm{mg} /$ $\mathrm{m}^{2}$. This course was repeated three weekly for a total of four courses. Response was evaluated with check cystoscopy and CT scan or MRI of the pelvis. If a complete response was observed, patients were offered CCRT. In case of less than complete response, radical cystectomy was offered to the patient. Patients declining radical cystectomy at this stage or patient deemed unfit for radical surgery due to co morbidity or age, were offered CCRT.

CCRT was carried out with weekly Cisplatin 40 $\mathrm{mg} / \mathrm{m}^{2}$. Radiation therapy to a dose of 64 Grays was administered in single phase to PTV that included bladder as $\mathrm{CTV} \pm 2 \mathrm{~cm}$ margin using three dimensional conformal radiotherapy (3D CRT) planning. In patients with involved pelvic lymph nodes, two-phased radiotherapy was executed with 44 Gy given to pelvis in phase I while 20 Gy was given in phase II to PTV that included bladder $\pm 2 \mathrm{~cm}$ margin. Response evaluation was again performed with MRI or CT scan of pelvis; and check cystoscopy 4-6 weeks after completion of CCRT.

Patients were placed on regular follow up. Check cystoscopy was repeated three monthly for 1st year, six monthly for 2nd and 3rd year and then yearly for next two years. Pelvic Imaging was repeated 6 monthly for 1 st two years and then yearly for next three years.

Responses were divided as per RECIST criteria10. Complete response was defined as disappearance of all disease. Partial response was defined as $30 \%$ or more decrease in tumor volume. Progressive disease was defined as $20 \%$ or more increase in tumor volume. Stable disease was defined as any response that does not fit the above categories. Primary study endpoints were 5 year survival and response evaluation at end of CCRT.

Overall survival was calculated using Kaplan Meier survival analysis through Statistical Package for the social sciences (SPSS) version 23.

\section{RESULTS}

A total of $112(92 \%)$ males and $10(8 \%)$ females with mean age of $56.69 \pm 7.6$ years (range 37-73) with muscle invasive bladder carcinoma, treated as per above mentioned protocol were evaluated. Out of 102 $(83.6 \%)$ of the total patient population had a positive history of smoking. Most common stage at presentation was T3N0, which was seen in $51(41.8 \%)$ patients. This was followed by T4N0 (42 patients (34.4\%), T2N0 (13 patients $(10.7 \%))$, T3N1 (8 patients $(6.5 \%), \mathrm{T} 2 \mathrm{~N} 1$ (5 patients $(4.1 \%))$ and $\mathrm{T} 4 \mathrm{~N} 1$ (3 patients $(2.5 \%))$. After first four courses of neoadjuvant chemotherapy, 88 patients $(72 \%)$ showed complete response, 20 (16.3\%) showed partial response, 9 (7.3\%) showed stable disease and $5(4.1 \%)$ showed progressive disease.

Twenty $(16.3 \%)$ patients who showed partial response were offered radical cystectomy but they either underwent radical chemoradiotherapy due to patient choice or advanced age or co morbid conditions that rendered the patient inoperable. After completion of CCRT, $93(76.2 \%)$ patients were having a complete response, $19(15.5 \%)$ had partial response, $5(4.1 \%)$ had stable disease and $5(4.1 \%)$ had progressive disease.

Patients were followed up for a mean time of $54.3 \pm 29.6$ months. Thirty two patients died during this time while 6 were lost to follow up. Twenty four deaths were registered due to muscle invasive bladder carcinoma while eight died due to other causes. Survival data shows 5-year overall survival to be $66 \%$. 
Kaplan-Meier survival curve for the whole population is shown in Figure-1.

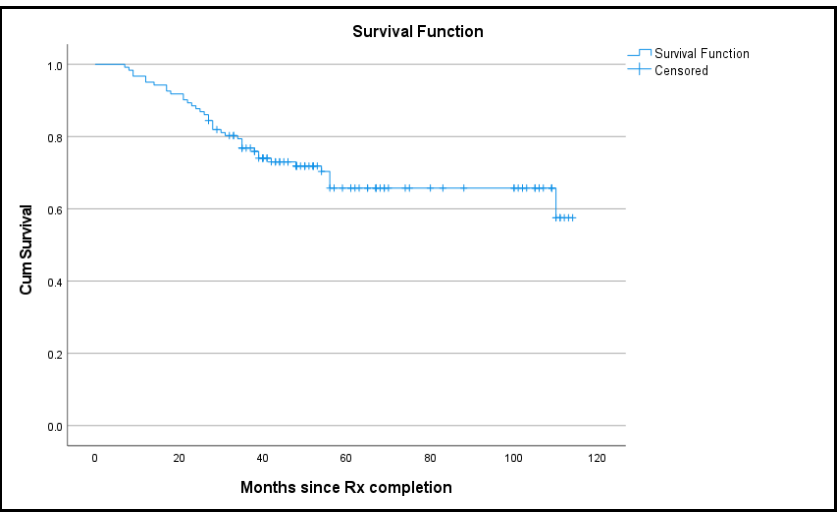

Figure-1: Kaplan-Meier survival curve for the whole study population.

Treatment was generally well tolerated by patients. Common side effects included mild to moderate nausea and vomiting ( $75 \%$ cases), urinary frequency $>5$ times a day during chemoradiation (70\% cases), reversible dysuria ( $80 \%$ cases), reversible strangury $(65 \%$ cases) and self-limiting haematuria ( $10 \%$ cases).

Kaplan-Meier Survival curve for each response group after CCRT is shown in Figure-2 which shows a markedly increased survival when a complete response is observed at the end of treatment.

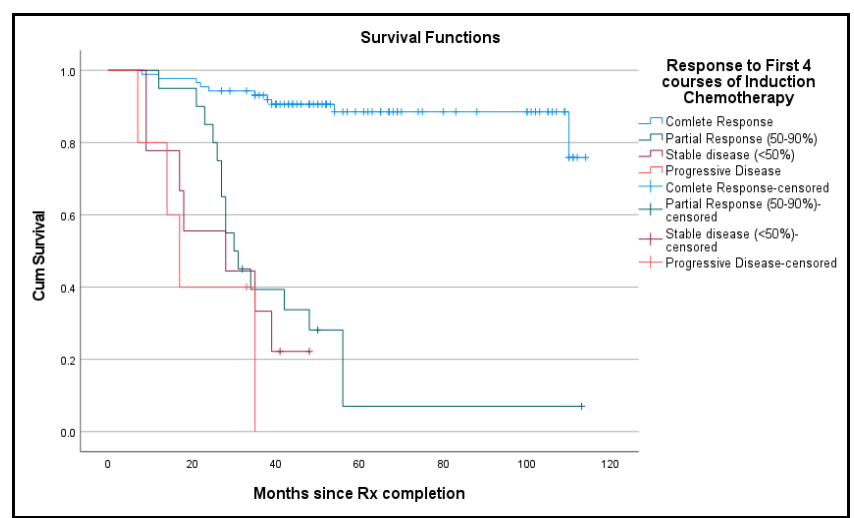

Figure-2: Kaplan-Meier survival curve for the subset according response to neoadjuvant chemotherapy

\section{DISCUSSION}

The multimodality management with maximal TURBT, followed by neoadjuvant chemotherapy and then definitive CCRT with weekly Cisplatin has shown CR in $93(76.2 \%)$ patients and the 5-year survival was $66 \%$ in this study. This has been compared with previous studies in the following table (Table) which evaluated the response of bladder preservation protocols.

As it can be observed from the Table; that the response rates at the end of therapy and the survival rate, are in line with the previous studies. ${ }^{11-14}$ Radical cystectomy has been the standard of care for treatment of Carcinoma of Urinary Bladder but it is not the kind of treatment that a patient would opt for if given a choice of bladder preservation approach with equivalent 5 year overall surviva. 12,15,16 If patient is offered bladder preservation protocol, he has the choice of salvage cystectomy which can be carried after confirmation of incomplete response or recurrent disease. Salvage cystectomy can be carried out within 12 weeks of radiotherapy completion to avoid extensive fibrosis during surgical manipulation of tissues. On the other hand, if the patient undergoes radical cystectomy and disease relapse occurs after some time, there is no option left except for palliative chemotherapy in majority of cases. Bladder preservation should be considered as a standard therapy in cases of muscle invasive bladder carcinoma at par with radical cystectomy with the advantage of organ preservation going in favor of bladder preservation protocol.

Table: Comparison of survival with historical control studies.

\begin{tabular}{|c|c|c|c|c|c|}
\hline $\begin{array}{l}\frac{\vec{C}}{2} \\
\text { के }\end{array}$ & 营 & 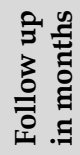 & 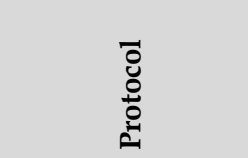 & 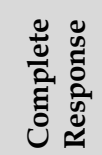 & 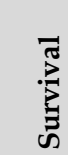 \\
\hline $\begin{array}{l}\text { Lin, et } \\
\text { al. }{ }^{11}\end{array}$ & 30 & 47 & $\begin{array}{c}\text { Neoadjuvant } \\
\text { CFx3 + paclitaxel, } \\
\text { ciplatin or } \\
\text { paclitaxel + RT }\end{array}$ & $73.3 \%$ & $77 \%$ \\
\hline $\begin{array}{l}\text { Kaufma, } \\
\text { et al. }{ }^{12}\end{array}$ & 53 & 48 & $\begin{array}{l}\text { Neoadjuvant } \\
\text { CMVx3, } \\
\text { cisplatin +RT }\end{array}$ & $58 \%$ & $48 \%$ \\
\hline $\begin{array}{l}\text { Tester } \\
\text { et al. }{ }^{13}\end{array}$ & 42 & 36 & $\begin{array}{c}\text { Concurrent } \\
\text { cisplatin+RT }\end{array}$ & $67 \%$ & $64 \%$ \\
\hline $\begin{array}{l}\text { Cervek } \\
\text { et al. }{ }^{14}\end{array}$ & 47 & 23 & $\begin{array}{c}\text { Neoadjuvant } \\
\text { CMVx3-4, } \\
\text { RT alone }\end{array}$ & $62 \%$ & $73 \%$ \\
\hline $\begin{array}{l}\text { Present } \\
\text { study }\end{array}$ & 122 & 49 & $\begin{array}{c}\text { Neoadjuvant } \\
\text { GCx4, Cisplatin } \\
\text { weekly + RT }\end{array}$ & $76.2 \%$ & $66 \%$ \\
\hline
\end{tabular}

The results in this study are better than the historical control studies of radical cystectomy 2 and comparable with the studies regarding bladder preservation. In addition, the overall survival was comparable to previous studies that have used this protocol of neoadjuvant chemotherapy followed by CCRT. However, the overall survival in this study is better than twoland mark studies i.e. BC2001 and BCON, long-term results of which have shown 5-year survival to be in the range of $39-45 \% .{ }^{17}$ A shortcoming in the study was the sample bias, as there is no control group. Further- 
more patients who were retrospectively analyzed included those patients too who were denied the option of cystectomy as a radical treatment; thus decreasing the overall survival of our patients. The reason for more proportion of $\mathrm{T} 3 \mathrm{~N} 0$ patients and less $\mathrm{T} 2 \mathrm{~N} 0$ patients is the selection bias towards radical cystectomy. Generally, fit patients having T2N0 disease are offered radical cystectomy as the only curative option and are never given an option of bladder preservation. With more patients of T2N0 undergoing bladder preservation, the response rates and hence overall survival can be improved even further. In addition, the post TURBT residual disease were not recorded in the study as this is considered to be a poor prognostic factor for bladder preservation. ${ }^{18}$

Prospective randomized studies rather than retrospective analysis are required to establish the fact that bladder preservation approach should be preferred over radical cystectomy or vice versa.

\section{LIMITATION OF STUDY}

No patient underwent salvage cystectomy after treatment failure as this study included only those patients who were deemed unfit for cystectomy at the time of initial diagnosis. However, the obvious limitation in the study was the absence of the simultaneous, randomized control group. Without this randomized control group, a sample bias cannot be eliminated.

\section{CONCLUSION}

Selective bladder preservation is a viable alternative to radical cystectomy when overall survival is seen as a final outcome measure. The cost of cystectomy without a continent diversion and its follow-up is very high when compared to bladder preservation protocol.

\section{Conflict of Interest: None.}

\section{Authors' Contribution}

ASS: Patient enrollment data maintenance, SH: Manuscript writing, data collection and analysis, FAS: Patient enrollment finalising manuscript.

\section{REFERENCES}

1. Tanaka H, Kijima T, Fujii Y. Bladder preservation therapy in muscle-invasive bladder cancer: Current evidence and future perspectives. AME Med J 2020; 5(1): 16-18.

2. Dalbagni G, Genega E, Hashibe M, Zhang Z-F, Russo P, Herr H, et al. Cystectomy for Bladder Cancer: A contemporary Series. J Urol 2001; 165(4): 1111-1116.

3. Lee YT, Wu YT, Yen CC, Chang MH, Chang YH, Chung HJ, et al. Concurrent chemoradiotherapy in elderly patients with muscleinvasive bladder cancer: A single-center experience. J Cancer Res Pract 2016; 3(3): 73-76.
4. Majewski W, Nieckula J, Dworzecki T, Miszczyk L. Bladderconserving Approach in Radical Treatment of Patients With Bladder Cancer - A Single-institution Experience. Anticancer Res 2020; 40(10): 5861-5868.

5. Ghate K, Brennan K, Karim S, Siemens DR, Mackillop WJ, Booth $\mathrm{CM}$. Concurrent chemoradiotherapy for bladder cancer: Practice patterns and outcomes in the general population. Radiother Oncol 2018; 127(1): 136-142.

6. Jiang DM, Jiang $\mathrm{H}$, Chung PWM, Zlotta AR, Fleshner NE, Bristow RG, et al. Neoadjuvant Chemotherapy Before BladderSparing Chemoradiotherapy in Patients With Nonmetastatic Muscle-Invasive Bladder Cancer. Clin Genitourin Cancer 2019; 17(1): 38-45.

7. Hussain SA, Porta N, Hall E, Huddart R, James ND. Outcomes In Patients In Muscle Invasive Bladder Cancer Treated With Neoadjuvant Chemotherapy Followed By (Chemo)Radiotherapy In The BC2001 Trail. Eur Urol 2021; 79(2): 307-315.

8. Ajib K, Tjong MC, Tan GH, Nason GJ, Berjaoui MB, Erlich A, et al. Canadian experience of neoadjuvant chemotherapy on bladder reccurrence in patients managed with trimodality therapy for muscle invasive bladder cancer. Can Urol Assoc J 2020; 14(12): 404-410.

9. Choueiri TK, Raghavan D. Chemotherapy for muscle-invasive bladder cancer treated with definitive radiotherapy: persisting uncertainties. Nat Clin Pract Oncol 2008; 5(8): 444-454.

10. Recist Working Group. Response Evaluation Criteria in Solid Tumors (version 1.1). Available at: https://recist.eortc.org/ recist-1-1-2/. [Accessed on December 12, 2020].

11. Lin CC, Hsu CH, Cheng JC, Huang CY, Tsai YC, Hsu FM, et al. Induction cisplatin and fluorouracil-based chemotherapy followed by concurrent chemoradiation for muscle-invasive bladder cancer. Int J Radiat Oncol Biol Phys 2009; 75(2): 442-448.

12. Kaufman DS, Shipley WU, Griffin PP, Heney NM, Althausen AF, Efird JT. Selective bladder preservation by combination treatment of invasive bladder cancer. N Engl J Med 1993; 329(19): 13771382.

13. Tester W, Porter A, Asbell S, Coughlin C, Heaney J, Krall J, et al. Combined modality program with possible organ preservation for invasive bladder carcinoma: Results of rtog protocol 85-12. Int J Radiat Oncol Biol Phys 1993; 25(5): 783-790.

14. Červek J, Čufer T, Kragelj B, Zakotnik B, Stanonik M. Sequential transurethral surgery, multiple drug chemotherapy and radiation therapy for invasive bladder carcinoma: Initial report. Int J Radiat Oncol Biol Phys 1993; 25(5): 777-782.

15. Tunio MA, Hashmi A, Qayyum A, Mohsin R, Sultan G. Outcome of Trimodality Protocol for invasive bladder cancer patients at Karachi, Pakistan. J Pak Med Assoc 2011; 61(9): 874-879.

16. Yafi FA, Kassouf W. Radical cystectomy is the treatment of choice for invasive bladder cancer. Can Urol Assoc J 2009; 3(5): 409-412.

17. Choudhury A, Port N, Hall E, Song YP, Owen R, Mackay R, et al. Hypofractionated radiotherapy in locally advanced bladder cancer: an individual patient data meta-analysis of the BC2001 and BCON trials. Lancet Oncol 2021; 22(2): 246-255.

18. Scrimger RA, Murtha AD, Parliament MB, Venner PM, Hanson J, Houle G, et al. Muscle-invasive transitional cell carcinoma of the urinary bladder: a population-based study of patterns of care and prognostic factors. Int J Radiat Oncol Biol Phys 2001; 51(1): 23-30. 